\title{
Influence of $\mathrm{SF}_{6}$ on the Broadening and Shift of the $535.0 \mathrm{~nm}$ Thallium Line*
}

\author{
E. Lisicki, R. Bobkowski, J. Szudy, and J. Wolnikowski \\ Institute of Physics, Nicholas Copernicus University, Toruń, Poland
}

Z. Naturforsch. 38a, 838-840 (1983); received March 4, 1983

\begin{abstract}
The pressure broadening and shift of the $535.0 \mathrm{~nm} \mathrm{Tl}$ line resulting from the photodissociation of thallium iodide mixed with sulfur hexafluoride were studied at low densities using a FabryPerot interferometer. A linear variation of both the width and shift of the line with the $\mathrm{SF}_{6}$ density was found and the cross sections for the broadening and shift were determined.
\end{abstract}

\section{Introduction}

In recent papers [1-3], studies of the Doppler and pressure broadening of the atomic fluorescence of thallium iodide perturbed by rare gas atoms and some non-polar molecules such as $\mathrm{H}_{2}, \mathrm{D}_{2}, \mathrm{~N}_{2}, \mathrm{O}_{2}$ as well as $\mathrm{CO}_{2}$ were reported. In these studies the values of the effective cross sections for the collision shift and broadening of the $535.0 \mathrm{~nm}\left(7^{2} \mathrm{~S}_{1 / 2}-6^{2} \mathrm{P}_{3 / 2}\right)$ atomic thallium fluorescence line resulting from the photodissociation of thallium iodide molecules were determined from the Lorentzian component of the line profile.

The present paper describes an extension of these investigations to measurements of collision effects on the same $\mathrm{Tl}$ line accompanying the photodissociation of TlI perturbed by sulfur hexafluoride $\left(\mathrm{SF}_{6}\right)$. This research is stimulated, in part, by recent work on collisions between Rydberg atoms and $\mathrm{SF}_{6}$ molecules which has shown that the cross sections for the excitation transfer between various fine structure levels were smaller than those observed when polar molecules have been used as perturbing gas but close to those caused by rare gas atoms $[4,5]$. Such results were not unexpected since $\mathrm{SF}_{6}$ has no permanent dipole moment and its mass and polarizability are comparable to those of the xenon atom. We have therefore thought it useful to study the broadening and shift effects on atomic thallium fluorescence produced by $\mathrm{SF}_{6}$ in order to compare them with those due to rare gas atoms, especially due to xenon.

* Work supported by the Committee of Spectroscopy of the Polish Academy of Sciences.

Reprint requests to Dr. E. Lisicki, Institute of Physics, Nicholas Copernicus University Toruń, Grudziadzka 5, 87-100 Toruń/Poland.

\section{Experimental}

The experimental procedure and the line shape analysis used in the present work were identical to those described in our previous papers on the pressure broadening and shift of the $535.0 \mathrm{~nm} \mathrm{Tl}$ line accompanying the photodissociation of thallium iodide $[1-3]$. The light was collected from the fluorescence cell containing TlI vapour with $\mathrm{SF}_{6}$. The photodissociation of TlI was excited by irradiation of the $\mathrm{Tl} \mathrm{I}_{-} \mathrm{SF}_{6}$ mixture with the ultraviolet light emitted from a radio-frequency mercury discharge lamp. The fluorescence cell was mounted in an electric oven, which could be maintained at any temperature between 300 and $750 \mathrm{~K}$ with stability of $\pm 2 \mathrm{~K}$ over several hours. The temperature was measured with a thermocouple placed in a contact with the fluorescence cell. Measurements reported in the present paper were carried out at the temperature of $733 \mathrm{~K}$ and at densities of sulfur hexafluoride up to $9 \times 10^{17} \mathrm{~cm}^{-3}$ which corresponds to a pressure of $\mathrm{SF}_{6}$ up to 16 Torr at room temperature.

Line profiles were analysed using a grating spectrograph and a pressure scanned Fabry-Perot etalon with $1.204 \mathrm{~cm}$ spacer and dielectric coating [6]. Our studies were performed on natural thallium which consists of $70.54 \%{ }^{205} \mathrm{Tl}$ and $29.46 \%{ }^{203} \mathrm{Tl}$. The two hyperfine-structure components determined by the splitting of the $7^{2} \mathrm{~S}_{1 / 2}$ level of $\mathrm{Tl}$ were resolved while the splitting due to the hyperfine-structure of $6^{2} \mathrm{P}_{3 / 2} \mathrm{Tl}$ level was not resolved by our interferometer. The methods of numerical analysis of the resultant profiles of overlapping line components were the same as in previous works [1-3]. In measurements of the collision shift of the centre of the $535.0 \mathrm{~nm} \mathrm{Tl}$ line a low pressure r.f. electrodeless discharge thallium lamp was used as a reference source. 


\section{Results and Discussion}

A very careful numerical analysis has shown that the resultant shape of the $535.0 \mathrm{~nm} \mathrm{Tl}$ line emitted due to the photodissociation of TlI can be sufficiently well approximated by the Voigt profile, which is the convolution of the Lorentzian and Gaussian profiles. The half-widths $\gamma_{L}$ and $\gamma_{D}$ of the Lorentzian and Gaussian components of the resultant profile, respectively, were determined using both a procedure given by Ballik [7] and a least squares method.

The results of our measurements are shown in Fig. 1, where the Gaussian and Lorentzian halfwidth, $\gamma_{\mathrm{D}}$ and $\gamma_{\mathrm{L}}$, of the $535.0 \mathrm{~nm} \mathrm{Tl}$ line as well as the shift $\Delta$ of this line are plotted against the sulfur hexafluoride density. It is seen that the Gaussian half-width is practically constant over the entire range of the $\mathrm{SF}_{6}$ density. The mean value $\bar{\gamma}_{\mathrm{D}}$ of the Gaussian half-width is equal to $0.052 \mathrm{~cm}^{-1}$ which is significantly greater than the Gaussian half-width $\gamma_{\mathrm{d}}=0.020 \mathrm{~cm}^{-1}$ corresponding to the usual Doppler broadening resulting from the thermal motion of $\mathrm{Tl}$ atoms with the Maxwellian velocity distribution at the temperature $733 \mathrm{~K}$. The difference between $\bar{\gamma}_{\mathrm{D}}$ and $\gamma_{\mathrm{d}}$ is due to the additional Doppler broadening arising from the recoil of the excited $\mathrm{Tl}\left(7^{2} \mathrm{~S}_{1 / 2}\right)$ atom after the photodissociation of TlI. It should be noted that the average Doppler width of the $535.0 \mathrm{~nm} \mathrm{Tl}$ line perturbed by $\mathrm{SF}_{6}$ at $733 \mathrm{~K}$ is close to the values found for this line perturbed by rare gas atoms and such non-polar molecules as $\mathrm{H}_{2}, \mathrm{D}_{2}$, $\mathrm{N}_{2}, \mathrm{O}_{2}$ and $\mathrm{CO}_{2}$ at the same temperature [1-3].

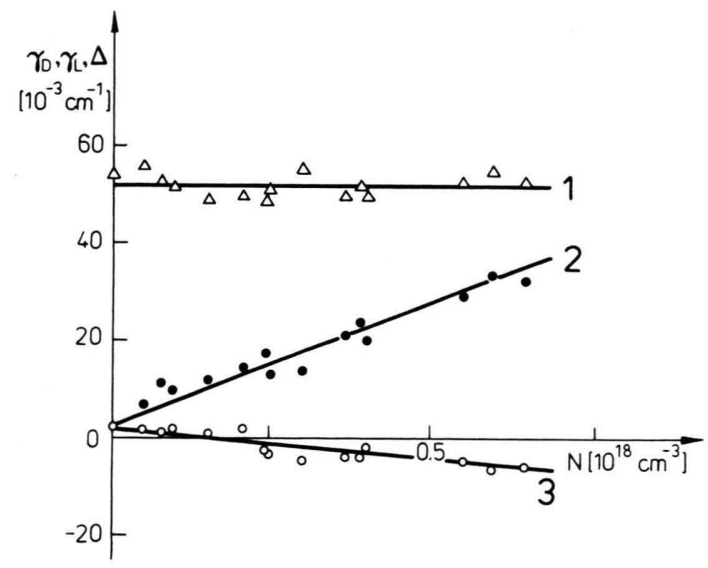

Fig. 1. Plots of the Gaussian (1), Lorentzian (2) half-widths and the shift (3) of the $535.0 \mathrm{~nm}$ Tl line against the density of sulfur hexafluoride. $\Delta O \bigcirc-$ experimental points.
As it is seen from Fig. 1 the Lorentzian (full) halfwidth $\gamma_{\mathrm{L}}$ of the $535.0 \mathrm{~nm}$ T1 line depends linearly on the density $N$ of sulfur hexafluoride according to the relation:

$$
\gamma_{\mathrm{L}}=\gamma_{0}+\beta N,
$$

where $\gamma_{0}$ is the asymptotic value of the Lorentzian half-width for the fluorescence cell with no perturbing gas and $\beta$ is the pressure broadening coefficient. Using the least-squares analysis we found for $\mathrm{Tl}+\mathrm{SF}_{6}$ the value $\beta=(5.12 \pm 0.47) \times 10^{-20} \mathrm{~cm}^{-1} /$ molecule $\mathrm{cm}^{-3}$. Let us note that in the case of the same line perturbed by Xe the measured value of the pressure broadening coefficient was found to be $\beta=(3.05 \pm 0.03) \times 10^{-20} \mathrm{~cm}^{-1} /$ atom $\mathrm{cm}^{-3}[2]$.

Figure 1 shows that the shift $\Delta$ of the $535.0 \mathrm{~nm} \mathrm{Tl}$ line is a linear function of the sulfur hexafluoride density:

$$
\Delta=\Delta_{0}+\delta N
$$

where $\Delta_{0}$ is the asymptotic shift for the TlI fluorescence cell without perturbing gas and $\delta$ is the pressure shift coefficient. From the least-squares analysis we found for $\mathrm{Tl}+\mathrm{SF}_{6}$ the value of the coefficient to be $\delta=(-1.59 \pm 0.30) \times 10^{-20} \mathrm{~cm}^{-1} /$ molecule $\mathrm{cm}^{-3}$. For the same line perturbed by Xe we have determined previously the value

$\delta=(-0.977 \pm 0.03) \times 10^{-20} \mathrm{~cm}^{-1} /$ molecule $\mathrm{cm}^{-3}$

[2]. Let us note that the shift is towards the red like in the case of perturbation by atoms of comparable polarizability (Xe, $\mathrm{Kr}$ ).

The red shift of the $535.0 \mathrm{~nm} \mathrm{Tl}$ line perturbed by $\mathrm{SF}_{6}$ is indicative of an attractive long-range potential for $\mathrm{Tl}+\mathrm{SF}_{6}$. The experimental value of the ratio of the shift to the Lorentzian half-width determined in the present work is equal to $\Delta / \gamma_{\mathrm{L}}=-0.311$. This value does not differ much from the theoretical value of this ratio $\Delta / \gamma_{\mathrm{L}}=-0.362$ calculated on the assumption that the interaction energy for $\mathrm{Tl}+\mathrm{SF}_{6}$ is given by the pure Van der Waals interaction [8].

Since our experimental values of the Lorentzian half-width $\gamma_{\mathrm{L}}$ and shift $\Delta$ are closely linear function of the $\mathrm{SF}_{6}$-density we can determine the cross-sections $\sigma_{\mathrm{b}}$ and $\sigma_{\mathrm{s}}$ for the pressure broadening and shift of the $535.0 \mathrm{~nm} \mathrm{Tl}$ line, respectively, using the impact approximation [8], according to which

$$
\beta=2 \bar{v} \sigma_{\mathrm{b}},
$$


and

$$
\delta= \pm \bar{v} \sigma_{\mathrm{s}} .
$$

Here $\bar{v}$ is the mean relative velocity of the colliding atom and molecule. Using the values $\beta$ and $\delta$ determined in the present investigation for $\bar{v}=6.4 \times$ $10^{4} \mathrm{~cm} / \mathrm{s}$ we found the values $\sigma_{\mathrm{b}}=7.5 \times 10^{-14} \mathrm{~cm}^{2}$ for the cross-section for the broadening and $\sigma_{\mathrm{s}}=4.6 \times$ $10^{-14} \mathrm{~cm}^{2}$ for the cross-section for the shift of the $535.0 \mathrm{~nm} \mathrm{Tl}$ line. These values of cross-sections are greater than those determined in our previous experiments on $\mathrm{Tl}$ perturbed by rare gas atoms, hydrogen, deuterium, oxygen, nitrogen and carbon dioxide. The reasonable agreement between our experimental data and theoretical values obtained for a Van der Waals interaction shows that in the first approximation the broadening and shift of the $535.0 \mathrm{~nm} \mathrm{Tl}$ line by $\mathrm{SF}_{6}$

[1] E. Lisicki, J. Szudy, and J. Wolnikowski, Acta Phys. Polon. A56, 557 (1979); A58, 105 (1980).

[2] E. Lisicki, A. Bielski, and J. Szudy, Z. Naturforsch. 35a, 1249 (1980); 37a, 93 (1982); 37a, 1094 (1982).

[3] E. Lisicki, A. Bielski, R. S. Dygdala, and J. Szudy, Acta Phys. Polon. A61, 421 (1982).

[4] F. G. Kellert, C. Higgs, K. A. Smith, G. H. Hildebrandt, F. B. Dunning, and R. F. Stebbings, J. Chem. Phys. 72, $6312(1980)$

are mainly due to purely adiabatic effects caused by the long range attractive forces between Tl-atom and $\mathrm{SF}_{6}$-molecule. However, there is a number of factors whose role should first be clarified before the final conclusions on the effects of the $\mathrm{SF}_{6}$ pressure on the atomic fluorescence of $\mathrm{Tl} I$ are drawn.

First of all, the quenching of the thallium $7^{2} S_{1 / 2}$ state by $\mathrm{SF}_{6}$ should be determined in order to estimate the influence of inelastic collisions on the $535.0 \mathrm{~nm}$ line. Secondly, reactive collisions between $\mathrm{SF}_{6}$ and the products of the photodissociation of thallium iodide which are possible may cause additional broadening effects superimposed on that resulting from the $\mathrm{Tl}^{-\mathrm{SF}_{6}}$ interaction. To clarify this problem studies of the pressure effects caused by $\mathrm{SF}_{6}$ on the fluorescence radiation emitted from the pure thallium vapour should be performed.

[5] F. B. Dunning and R. F. Stebbings, in: Rydberg Atoms, eds. R. F. Stebbings and F. B. Dunning, Cambridge University Press, Cambridge 1982.

[6] A. Bielski, W. Dokurno, E. Lisicki, and Z. Turlo, Opt. Appl. 11, 151 (1981).

[7] E. A. Ballik, Appl. Opt. 5, 170 (1966).

[8] I. I. Sobelman, Introduction to the Theory of Atomic Spectra, Pergamon Press, Oxford 1972. 